\title{
A aplicação da musicoterapia na doença de Parkinson: uma revisão integrativa
}

\author{
The application of music therapy in Parkinson's disease: an integrative review
}

La aplicación de la musicoterapia en la enfermedad de Parkinson: una revisión integradora

Luzia de Oliveira Chaves $^{1 *}$, Philip George Glass Andrade

\section{RESUMO}

Objetivo: Revisar na literatura a aplicação da musicoterapia como terapia adjuvante na Doença de Parkinson (DP). Métodos: Pesquisa exploratória e qualitativa, desenvolvida através da técnica de revisão de literatura integrativa, realizada por meio do banco de dados PUBMED, LILAC, MEDLINE, SCIELO e ACERVO + INDEX BASE. As palavras chave de busca utilizadas fora: "music therapy" e "Parkinson disease". Dos 124 artigos apontados pela busca, 15 foram selecionados para o presente estudo considerando critérios de inclusão e exclusão previamente definidos. Os dados dos estudos selecionados foram organizados em tabela para comparação os resultados encontrados. Resultados: Dentre o universo amostral analisado analisou-se a influência da musicoterapia na motricidade, qualidade de vida, humor, cognição, inteligibilidade e padrões acústicos de fala em pacientes com DP, utilizando diversas ferramentas confiáveis, validas e sensíveis. Os dados encontrados corroboram pelo uso da musicoterapia no manejo da DP de forma a complementar a atual terapêutica farmacologia e promover uma melhor qualidade de vida para esses pacientes. Considerações finais: Impera-se a produção de mais ensaios clínicos randomizados, para corroborar tais dados e avaliar se esses efeitos se estendem a longo prazo e a pacientes com DP grave.

Palavras-chave: Doença de Parkinson, Musicoterapia, Dança, Canto, Reabilitação vocal.

\begin{abstract}
Objective: To review in the literature the application of music therapy as adjuvant therapy in Parkinson's Disease (PD). Methods: Exploratory and qualitative research, developed through the technique of integrative literature review, carried out through the database PUBMED, LILAC, MEDLINE, SCIELO and ACERVO + INDEX BASE. The search keywords used were: "music therapy" and "Parkinson disease". Of the 124 articles identified by the search, 15 were selected for this study considering previously defined inclusion and exclusion criteria. Data from selected studies were organized in a table to compare the results found. Results: Among the sample universe analyzed, the influence of music therapy on motricity, quality of life, mood, cognition, intelligibility and acoustic patterns of speech in PD patients were analyzed, using several reliable, valid and sensitive tools. The data found corroborate the use of music therapy in the management of PD in order to complement the current pharmacological therapy and to promote a better quality of life for these patients. Final considerations: The production of more randomized clinical trials is imperative to corroborate these data and assess whether these effects extend to the long term and to patients with severe PD.
\end{abstract}

Keywords: Parkinson's disease, Music therapy, Dancing, Singing, Vocal rehabilitation.

\section{RESUMEN}

Objetivo: Revisar en la literatura la aplicación de la musicoterapia como terapia adyuvante en la Enfermedad de Parkinson (EP). Métodos: Investigación exploratoria y cualitativa, desarrollada mediante la técnica de revisión integrativa de la literatura, realizada a través de la base de datos PUBMED, LILAC, MEDLINE, SCIELO y ACERVO + INDEX BASE. Las palabras clave de búsqueda utilizadas fueron: "musicoterapia" y "enfermedad de Parkinson". De los 124 artículos identificados por la búsqueda, 15 fueron seleccionados para este estudio considerando criterios de inclusión y exclusión previamente definidos. Los datos de los estudios seleccionados se organizaron en una tabla para comparar los resultados encontrados. Resultados: Entre el universo muestral analizado, se analizó la influencia de la musicoterapia en la motricidad, la calidad de vida, el estado de ánimo, la cognición, la inteligibilidad y los patrones acústicos del habla en pacientes con EP, utilizando varias herramientas confiables, válidas y sensibles. Los datos encontrados corroboran el uso de la musicoterapia en el manejo de la EP con el fin de complementar la terapia farmacológica actual y promover una mejor calidad de vida de estos pacientes. Consideraciones finales: La producción de más ensayos clínicos aleatorizados es imperativa para corroborar estos datos y evaluar si estos efectos se extienden a largo plazo y a los pacientes con EP grave.

Palabras clave: Enfermedad de Parkinson, Musicoterapia, Baile, Canto, Rehabilitación vocal.

${ }^{1}$ Universidade Estadual do Sudoeste da Bahia (UESB), Vitória da Conquista - BA. *E-mail: ziziachaves@hotmail.com 


\section{INTRODUÇÃO}

Descrita inicialmente em 1817 por James Parkinson como "paralisia agitante", a doença de Parkinson (DP) é uma doença crônica degenerativa progressiva do sistema nervoso central caracterizada, patologicamente, por degeneração dos neurônios dopaminérgicos da substância negra e, clinicamente, por sintomas motores, autonômicos e cognitivos. Trata-se da doença neurodegenerativa de segunda maior prevalência, com cerca de 100 a 200 casos por 100000 habitantes, com distribuição universal, atingindo todas as etnias e grupos socioeconômicos (RIEDER RM, et al., 2010).

Pacientes com DP constituem grupo heterogêneo. Além de dois dos três sintomas motores clássicos essenciais para o diagnóstico da doença (bradicinesia, rigidez e tremor), que causam diversos sinais e sintomas motores secundários (como a marcha Parkinsoniana e disartria), o portador da doença poderá apresentar alterações sensitivas e disautonômicas, movimentos involuntários (principalmente associado ao uso da levodopa) sintomas neuropsiquiátricos, e outros sintomas como hiposmia, que podem inclusive preceder o surgimento dos sintomas motores (WERNECK AL, 2010). Tais modificações sistêmicas culminam na necessidade por parte do paciente de adaptação à vida com a DP, associada tanto à realização das Atividades de Vida Diárias (AVD), quanto nas relações pessoais e interpessoais (VALCARENGHI RV, et al., 2018).

O tratamento da doença torna-se complexo ao considerar diversos fatores: natureza progressiva da DP; diversidade de sinais e sintomas; efeitos adversos das intervenções terapêuticas atualmente disponíveis; e ausência de terapia neuroprotetora e curativa. Assim, o tratamento atual resume-se a drogas que, por meio do aumento do estímulo dopaminérgico e/ou diminuição do estímulo colinérgico/glutamatérgico, proporcionam melhora parcial da sintomatologia do paciente, às custas de: discinecias, flutuações motoras, complicações neuropsiquiátricas, necessidade de doses cada vez maiores e sintomas cada vez mais refratários à terapia (RIEDER RM, et al., 2010; WERNECK AL, 2010).

Vistas à limitação das opções terapêuticas disponíveis, o emprego de tratamentos não farmacológicos como a musicoterapia pode ser grande auxílio no manejo dos sintomas e melhora qualidade de vida (SANTOS FR e CORONAGO VM, 2017; CÔRTE B e LODOVICI NETO B, 2009). Usada como recurso terapêutico, música pode promover alterações físicas, mentais e sociais que repercutirão na recuperação e reposta ao tratamento de diversas patologias (OLIVEIRA MF, et al., 2014). O uso da musicoterapia, com uso de movimentos rítmicos, proporciona uma melhora global da mobilidade em pacientes com DP (POHL P, et al., 2013). Ao sincronizar o treino de marcha com a música, a musicoterapia melhorou aspectos da marcha dos pacientes com DP, como a velocidade e cadência de passos, além da uma diminuição dos sintomas motores primários (HU B, et al., 2010).

A musicoterapia é campo de pesquisa ascendente. Apesar da música ser utilizada empiricamente como terapia desde os tempos remotos, como demonstrado em relatos bíblicos, gregos e romanos, apenas em meados do século XX, a fusão dessas áreas estabeleceu-se sistematicamente como prática na medicina. Trata-se de campo transdisciplinar, com fronteiras difíceis de definir, aplicações variadas, submetido a influencias culturais e temporais, ainda em desenvolvimento (BRUSCIA KE, 2016). Define-se, para fins teóricos e de síntese, proposta por Bruscia KE (2016) como processo reflexivo, no qual o terapeuta ajuda o cliente a melhorar sua saúde em experiências musicais e suas relações derivadas.

Trata-se de potencial estratégia terapêutica coadjuvante em diversas patologias, com efeitos positivos na recuperação, reabilitação e sintomatologia de pacientes com AVC prévio, demência, DP, epilepsia e esclerose múltipla. Apesar de não estar esclarecido o mecanismo exato pelo qual a música interviria em tais patologias, diversas hipóteses foram levantadas, como ativação neuronal e neuroplasticidade, ativação de redes de recompensa, excitação e emocionais, e ativação de redes neurais alternativas (SIHVONEN AJ, et al., 2017).

Ademais, intensa resposta emocional a música é capaz de ativar sistema mesolimbico dopaminérgico, causando melhora do humor, atenção, memória, motivação e função executiva; ativar o sistema nervoso parassimpático e inibir o sistema nervoso simpático. Finalmente, forte interação entre o sistema auditivo e sistema motor poderia explicar os efeitos positivos da música em pacientes com disfunção do sistema motor (SIHVONEN AJ, et al., 2017). 
Nesse contexto questiona-se: A musicoterapia é uma alternativa viável como terapia adjuvante no tratamento da Doença de Parkinson? Assim, a presente pesquisa tem como objetivo geral apresentar uma revisão dos ensaios clínicos que avaliaram a eficiência da musicoterapia em pacientes com Doença de Parkinson.

\section{MÉTODOS}

Pesquisa exploratória e qualitativa, desenvolvida através da técnica de revisão de literatura, realizada por meio do banco de dados PUBMED, LILAC, MEDLINE, SCIELO e ACERVO + INDEX BASE. As palavraschave de busca utilizadas foram: "music therapy", "Parkinson disease".

Considerando-se os artigos completos e publicados nos últimos 5 anos, a busca aponta para 124 artigos (PUBMED 37, LILAC 6, ACERVO + INDEX BASE 0, MEDLINE 78, SCIELO 3), sendo incluídos no trabalho apenas os que cumpriam os seguintes critérios de ensaios clínicos: a) relacionados a aplicação da musicoterapia como terapia adjuvante na Doença de Parkinson; b) descritivos dos métodos de musicoterapia utilizados na doença de Parkinson; c) descritivos do impacto da musicoterapia nos desfechos motores da doença; d) descritivos do impacto da musicoterapia nos desfechos não motores da doença; e) descritivos do impacto da musicoterapia na qualidade de vida dos pacientes com doença de Parkinson.

Excluiu-se os artigos de: a) ensaios clínicos descrevendo o uso de musicoterapia em outras doenças neurodegenerativas que não a Doença de Parkinson; b) ensaios clínicos descrevendo outras terapias alternativas no tratamento da Doença de Parkinson, que não a musicoterapia; c) relatos de caso, séries de caso, artigos epidemiológicos e de revisão (Figura 1).

Os ensaios clínicos foram colocados em tabela com autor, ano, número de participantes, participação do musicoterapeuta, desenho de estudo, intervenção realizada, resultados primários, duração da intervenção e principais resultados objetivando comparar os resultados encontrados. Os 15 artigos selecionados estavam na língua inglesa, e foram publicados entre os anos de 2016 e 2021.

Figura 1 - Processo de seleção dos artigos utilizados no presente estudo.

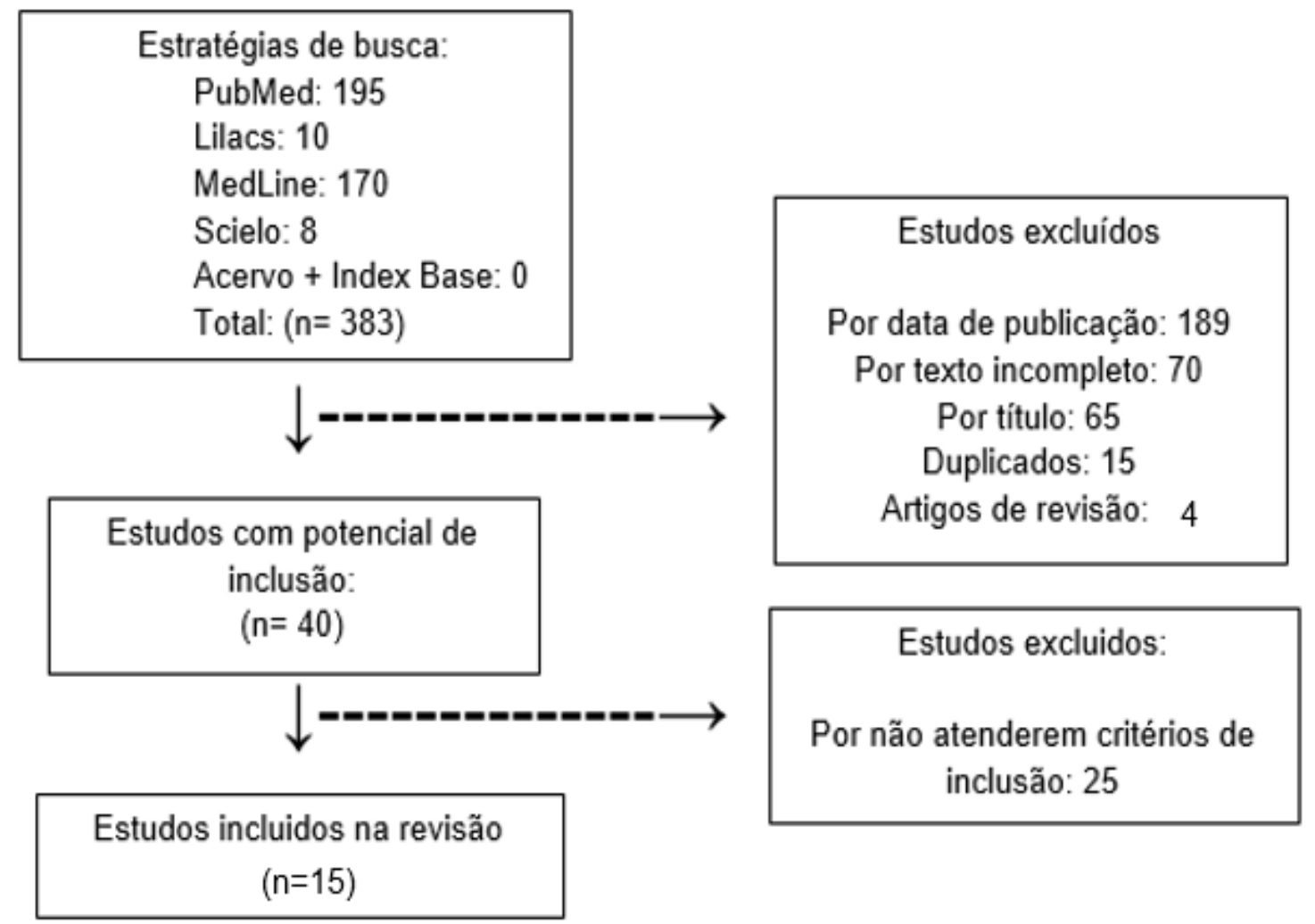

Fonte: Chaves LO e Andrade PG, 2021. 


\section{RESULTADOS E DISCUSSÃO}

Os ensaios analisaram a influência da musicoterapia na motricidade, qualidade de vida, humor e cognição, e inteligibilidade e padrões acústicos de fala em pacientes com DP.

Tais variáveis foram analisadas utilizando ferramentas confiáveis, validas e sensíveis para análise de pacientes com DP, como testes motores padrão (avaliação do nível de déficit motor geral com a Escala Unificada de Avaliação para Doença de Parkinson UPDRS-3; estadiamento de gravidade da DP com a escala dos estágios de incapacidade de Hoehn e Yahr; mobilidade com a Timed-Up-and-Go (TUG), questionário de freezing, GAITRite walkway; tolerância funcional ao exercício com o 6-minuts walk test; equilíbrio com a escala de equilíbrio de Berg), teste de capacidade cognitiva (Cognitive Assessment Battery-CAB e MiniMental test), e questionário de auto percepção de qualidade de vida (PDQ-39).

Um dos ensaios clínicos foi randomizado. Todos utilizaram pacientes com DP na ausência de déficits, auditivos e visuais relevantes diagnosticados. Apenas um analisou pacientes com déficits cognitivos; um analisou DP grave; aceitou pacientes com formas atípicas de DP. Ademais, os pacientes foram orientados a continuarem suas rotinas diárias e de exercícios e a não alterarem as medicações em uso em todos os estudos (Quadro 1). 
Quadro 1 - Análise de dados dos artigos selecionados para o presente estudo.

\begin{tabular}{|c|c|c|c|c|c|c|c|}
\hline Autor/Ano & $\begin{array}{c}\text { Participantes } \\
\text { (n) }\end{array}$ & $\begin{array}{c}\text { Método de } \\
\text { intervenção }\end{array}$ & $\begin{array}{l}\text { Participação do } \\
\text { musicoterapeuta }\end{array}$ & Randomização & Resultados primário & $\begin{array}{l}\text { Duração da } \\
\text { intervenção }\end{array}$ & Principais resultados \\
\hline $\begin{array}{l}\text { Calabro RS, } \\
\text { et al. (2019) }\end{array}$ & 50 & $\begin{array}{c}\text { Estímulo } \\
\text { Auditivo } \\
\text { Rítmico (RAS) } \\
\text { (MP) }\end{array}$ & Não & Único cego & $\begin{array}{l}\text { Mobilidade, equilíbrio, } \\
\text { parâmetros de } \\
\text { marcha, alterações no } \\
\text { Eletroencefalograma } \\
\text { (ECG) }\end{array}$ & $\begin{array}{l}\text { 195min/dia, } 5 x \\
\text { por semana, } \\
\text { durante } 8 \\
\text { semanas }\end{array}$ & $\begin{array}{l}\text { Os dados sugerem uma reformulação dos ritmos } \\
\text { sensório-motores e da conectividade fronto- } \\
\text { centroparietal/temporal dependente de uma contribuição } \\
\text { cerebelar com o treino RAS. Ademais, houve maior } \\
\text { melhora na mobilidade, equilíbrio e parâmetros de } \\
\text { marcha com o treino RAS. }\end{array}$ \\
\hline $\begin{array}{l}\text { Bella SD, et } \\
\text { al. (2017) }\end{array}$ & 14 & RAS (MP) & Não & Único cego & $\begin{array}{l}\text { Parâmetros de marcha } \\
\text { e capacidade de } \\
\text { sincronização da } \\
\text { marcha com batidas } \\
\text { de palmas. }\end{array}$ & 4 semanas & $\begin{array}{l}\text { A resposta ao RAS foi variável, com alguns pacientes } \\
\text { respondendo positivamente (com aumento da velocidade } \\
\text { de marcha e comprimento de passo), outras } \\
\text { negativamente e outros não apresentando resposta } \\
\text { alguma. Os resultados corroboram a ideia de que as } \\
\text { habilidades sensoriomotoras de temporização que } \\
\text { sustentam a sincronização de passos com as pistas } \\
\text { auditivas podem prever o sucesso do RAS na DP. Com } \\
\text { base no desempenho nas tarefas de batidas de palmas e } \\
\text { marcha foi possível prever uma resposta positiva ao RAS, } \\
\text { sendo o comprometimento da marcha mais grave, a baixa } \\
\text { variabilidade de sincronização e uma resposta imediata a } \\
\text { uma mudança de estimulação indicadores de tal resposta. }\end{array}$ \\
\hline $\begin{array}{l}\text { Thaut } \mathrm{MH} \text {, et } \\
\text { al. (2019). }\end{array}$ & 60 & RAS (MP) & Não & Único cego & $\begin{array}{c}\text { Parâmetros clínicos e } \\
\text { cinemáticos de } \\
\text { marcha. }\end{array}$ & $\begin{array}{l}\text { Treinos diários } \\
\text { de } 30 \text { min } \\
\text { durante } 24 \\
\text { semanas, } \\
\text { sendo que o } \\
\text { grupo controle } \\
\text { interrompeu o } \\
\text { treino RAS } \\
\text { entre a } 6^{\text {a }} \text { e } \\
16^{\text {a }} \text { semanas. }\end{array}$ & $\begin{array}{l}\text { Ambos os grupos apresentaram melhoras significativas } \\
\text { na } 8^{\mathrm{a}} \text { semana, entretanto, mas na } 16^{\mathrm{a}} \text { semana surgiram } \\
\text { diferenças significativas entre os grupos (incluindo } \\
\text { aumento do índice de queda para o grupo controle), que } \\
\text { já não eram mais significativas na } 24^{\underline{a}} \text { semana. Além } \\
\text { disso, as mudanças na marcha, medo de cair e índice de } \\
\text { queda correlacionaram-se significativamente com a } \\
\text { dorsiflexão bilateral do tornozelo, indicando a mesma } \\
\text { como potencial mecanismo cinemático que o RAS aborda } \\
\text { para reduzir as quedas. }\end{array}$ \\
\hline $\begin{array}{l}\text { Fodor DM, et } \\
\text { al. (2021) }\end{array}$ & 32 & $\begin{array}{l}\text { Ouvir música } \\
(\mathrm{MP})\end{array}$ & Não & Único Cego & Qualidade de vida & $\begin{array}{l}2,5 \text { h diárias } \\
\text { por } 14 \text { dias. }\end{array}$ & $\begin{array}{l}\text { O grupo de estudo registrou maiores melhorias em cinco } \\
\text { das oito áreas da vida avaliadas pelo PDQ- } 39 \text { em } \\
\text { comparação com o grupo de controle, sugerindo que ouvir } \\
\text { música combinado com um programa de reabilitação } \\
\text { multimodal centrado em fisioterapia pode ser benéfica } \\
\text { para a qualidade de vida na DP. }\end{array}$ \\
\hline
\end{tabular}




\begin{tabular}{|c|c|c|c|c|c|c|c|}
\hline Autor/Ano & $\begin{array}{l}\text { Participantes } \\
\text { (n) }\end{array}$ & $\begin{array}{l}\text { Método de } \\
\text { intervenção }\end{array}$ & $\begin{array}{l}\text { Participação do } \\
\text { musicoterapeuta }\end{array}$ & Randomização & Resultados primário & $\begin{array}{l}\text { Duração da } \\
\text { intervenção }\end{array}$ & Principais resultados \\
\hline $\begin{array}{l}\text { Chomiak T, } \\
\text { et al. (2017) }\end{array}$ & 11 & $\begin{array}{l}\text { Ambulosono } \\
\text { (MP) }\end{array}$ & Não & Não & $\begin{array}{l}\text { Automaticidade do } \\
\text { passo, medo de } \\
\text { queda, cognição e } \\
\text { congelamento de } \\
\text { marcha } \\
\end{array}$ & 4 semanas & $\begin{array}{l}\text { Foi observada melhora global na automaticidade de } \\
\text { passos em contextos de dupla-tarefa. Não houveram } \\
\text { mudanças nos outros parâmetros. }\end{array}$ \\
\hline $\begin{array}{l}\text { Burt J, et al. } \\
\quad(2020)\end{array}$ & 30 & $\begin{array}{l}\text { Ambulosono } \\
\text { (MP) }\end{array}$ & Não & Sim & $\begin{array}{l}\text { Cognição, humor e } \\
\text { ansiedade }\end{array}$ & $\begin{array}{l}12 \text { semanas } \\
\text { para o grupo } \\
\text { de } \\
\text { intervenção. } 6 \\
\text { semanas para } \\
\text { o grupo } \\
\text { controle. }\end{array}$ & $\begin{array}{l}\text { Apesar de as melhorias nas medidas cognitivas não } \\
\text { terem sido clinicamente significativas, diminuições } \\
\text { significativas na depressão e ansiedade foram } \\
\text { observados em ambos os grupos ao longo do tempo, } \\
\text { demonstrando que o treinamento de marcha contingente } \\
\text { à música é viável e seguro em indivíduos com DP. }\end{array}$ \\
\hline $\begin{array}{l}\text { Spina E, et } \\
\text { al. }(2016)\end{array}$ & 25 & $\begin{array}{l}\text { Música, canto e } \\
\text { dança (MT) }\end{array}$ & Sim & Único cego & $\begin{array}{l}\text { Cognição, qualidade } \\
\text { de vida e sintomas } \\
\text { motores. }\end{array}$ & $\begin{array}{l}\text { Sessões } \\
\text { semanais de } \\
\text { 90min durante } \\
24 \text { semanas. }\end{array}$ & $\begin{array}{l}\text { Foram encontrados efeitos positivos na cognição e na } \\
\text { qualidade de vida, entretanto não foram mantidos } 6 \\
\text { meses após o fim da intervenção. Os sintomas motores } \\
\text { não se alteraram durante a intervenção, mas houve } \\
\text { deterioração entre o fim da intervenção e após } 6 \text { meses, } \\
\text { refletindo um possível efeito benéfico na marcha e } \\
\text { estabilidade. }\end{array}$ \\
\hline $\begin{array}{l}\text { Shanahan J, } \\
\text { et al. (2017). }\end{array}$ & 90 & Dança (MT) & Não & Único cego & Viabilidade & $\begin{array}{l}25 \mathrm{~h} \text { em } 10 \\
\text { semanas }\end{array}$ & $\begin{array}{l}\text { O estudo mostrou-se viável. Após a intervenção, uma } \\
\text { tendência a significância foi observada na UPDRS-3, uma } \\
\text { vez que o grupo de cuidados usuais apresentou piora na } \\
\text { UPDRS-3 e o grupo de dança apresentou leve melhora. }\end{array}$ \\
\hline $\begin{array}{l}\text { Tilmann AC, } \\
\text { et al. (2020). }\end{array}$ & 20 & Dança (MT) & Não & Não & Viabilidade & $\begin{array}{l}\text { Aulas de } 1 \mathrm{~h}, \\
2 \mathrm{x} \text { na semana } \\
\text { durante } 12 \\
\text { semanas }\end{array}$ & $\begin{array}{l}\text { O grupo experimental teve melhorias no UPDRS, } \\
\text { atividades diárias, exame motor, escores de equilíbrio, } \\
\text { escores de mobilidade e no domínio da qualidade de vida } \\
\text { no grupo experimental, de forma que o protocolo do } \\
\text { samba parece ser viável e seguro para pacientes com PD. } \\
\text { Ademais houveram beneficios nas relações sociais. }\end{array}$ \\
\hline $\begin{array}{l}\text { Harrison EC, } \\
\text { et al. (2019). }\end{array}$ & 60 & Canto (MT) & Não & Não & $\begin{array}{l}\text { Parâmetros e } \\
\text { variabilidade de } \\
\text { marcha. }\end{array}$ & $\begin{array}{c}\text { Estudo } \\
\text { transversal. }\end{array}$ & $\begin{array}{l}\text { Todos os participantes melhoraram a cadência e } \\
\text { comprimento da passada durante, entretanto pistas } \\
\text { externas resultaram em aumento da variabilidade da } \\
\text { marcha, enquanto as pistas internas diminuíram a mesma } \\
\text { em relação à caminhada sem pistas (principalmente } \\
\text { durante o canto mental). }\end{array}$ \\
\hline $\begin{array}{l}\text { Han EY, et } \\
\text { al. (2018) }\end{array}$ & 9 & Canto (MT) & Sim & Não & $\begin{array}{l}\text { Parâmetros de marcha } \\
\text { e depressão. }\end{array}$ & $\begin{array}{l}6 \text { sessões } \\
\text { durante } 2 \\
\text { semanas }\end{array}$ & $\begin{array}{l}\text { Houve melhora significativa no tempo máximo de fonação, } \\
\text { no Índice de desvantagem de voz e na escala de } \\
\text { depressão geriátrica, sendo que as melhoras no MPT, } \\
\text { GDS e qualidade de vida relacionada a voz mantiveram- } \\
\text { se após } 6 \text { meses do fim da intervenção. }\end{array}$ \\
\hline
\end{tabular}




\begin{tabular}{|c|c|c|c|c|c|c|c|}
\hline Autor/Ano & $\begin{array}{c}\text { Participantes } \\
\text { (n) }\end{array}$ & $\begin{array}{c}\text { Método de } \\
\text { intervenção }\end{array}$ & $\begin{array}{l}\text { Participação do } \\
\text { musicoterapeuta }\end{array}$ & Randomização & Resultados primário & $\begin{array}{l}\text { Duração da } \\
\text { intervenção }\end{array}$ & Principais resultados \\
\hline $\begin{array}{l}\text { Harrison EC, } \\
\text { et al. (2018) }\end{array}$ & 23 & Canto (MT) & Não & Não & Viabilidade & $\begin{array}{l}\text { Estudo } \\
\text { transversal }\end{array}$ & $\begin{array}{l}\text { Os resultados sugerem que o canto é uma opção viável } \\
\text { como técnica de pista auditiva na DP. Cantar enquanto } \\
\text { caminhava não afetou negativamente a marcha, } \\
\text { indicando que não houve demanda cognitiva excessiva } \\
\text { como na dupla tarefa. Ademais houve menor variabilidade } \\
\text { da marcha durante o canto com marcha. }\end{array}$ \\
\hline $\begin{array}{l}\text { Tamplin J, et } \\
\text { al. (2019). }\end{array}$ & 75 & Canto (MT) & Sim & Não & $\begin{array}{l}\text { Intensidade vocal, } \\
\text { inteligibilidade de fala, } \\
\text { comprimento máximo } \\
\text { de fonação, qualidade } \\
\text { de vida relacionada a } \\
\text { voz e pressões } \\
\text { respiratórias máximas. }\end{array}$ & 3 meses & $\begin{array}{l}\text { O grupo de intervenção apresentou melhorias } \\
\text { significativas na intensidade vocal, pressão expiratória } \\
\text { máxima, e qualidade de vida relacionada à voz em } \\
\text { comparação aos controles, não havendo diferenças no } \\
\text { comprimento máximo de fonação ou pressão inspiratória } \\
\text { máxima. Ademais houve efeito de dose, com o grupo } \\
\text { semanal apresentando melhora mais intensa. Em } \\
\text { contraste, a intensidade vocal diminuiu nos grupos de } \\
\text { controle sem tratamento. }\end{array}$ \\
\hline $\begin{array}{l}\text { Harrison EC, } \\
\text { et al. (2020) }\end{array}$ & 35 & MP e MT & Não & Não & $\begin{array}{c}\text { Trajetória dos } \\
\text { movimentos dos } \\
\text { membros inferiores }\end{array}$ & $\begin{array}{l}\text { Estudo } \\
\text { transversal }\end{array}$ & $\begin{array}{l}\text { Pacientes que respondem positivamente as pistas } \\
\text { auditivas usaram ambos os tipos de dicas (externas e } \\
\text { autogeradas) para aumentar a velocidade da marcha e } \\
\text { induzir aumentos na amplitude de movimento (ADM) das } \\
\text { articulações no quadril, enquanto apenas as dicas } \\
\text { autogeradas também aumentaram a ADM no } \\
\text { tornozelo. Ademais os respondedores apresentaram } \\
\text { maiores excursões conjuntas na fase de apoio e balanço. }\end{array}$ \\
\hline $\begin{array}{l}\text { Pohl P, et al. } \\
\qquad(2020)\end{array}$ & 46 & $\begin{array}{c}\text { Método Ronnie } \\
\text { Gardiner (MP e } \\
\text { MT) }\end{array}$ & Sim & Sim & $\begin{array}{l}\text { Habilidade de dupla } \\
\text { tarefa, cognição, } \\
\text { equilíbrio, } \\
\text { preocupação com } \\
\text { quedas, congelamento } \\
\text { de marcha e qualidade } \\
\text { de vida }\end{array}$ & 12 semanas & $\begin{array}{l}\text { Não foram observadas diferenças entre os grupos para a } \\
\text { habilidade de dupla tarefa. Apesar de não terem sido } \\
\text { mantidas após } 3 \text { meses de intervenção, Diferenças entre } \\
\text { grupos foram observados para Escala de Eficácia de } \\
\text { Quedas e para os itens do PDQ-39 quando comparado } \\
\text { ao grupo controle pós-intervenção. Ademais, os } \\
\text { resultados relatados pelo paciente e as entrevistas } \\
\text { sugerem que a intervenção musical baseada em grupo } \\
\text { adiciona valor para o humor, estado de alerta e qualidade } \\
\text { de vida em pacientes com doença de } \\
\text { Parkinson. Entretanto, os resultados não apoiam a } \\
\text { eficácia na produção de ganhos imediatos ou duradouros } \\
\text { em dupla tarefa, cognição, equilíbrio ou congelamento da } \\
\text { marcha. }\end{array}$ \\
\hline
\end{tabular}

Fonte: Chaves LO e Andrade PG, 2021. 


\section{Musicoterapia Passiva}

O uso de Estímulos rítmicos auditivos (RAS) mostra-se como promissora estratégia de reabilitação motora em pacientes com DP. Diversas alterações de marcha presentes em pacientes com DP correlacionam-se diretamente com perda de automaticidade e ritmicidade do movimento associada aos níveis deficientes de dopamina a nível da rede motora cortico-estriatal e consequente comprometimento dos mecanismos cerebrais geradores do ritmo de caminhada regular (CALABRÒ RS, et al., 2019).

Assim, pistas auditivas externas individualizadas indicadas pela cadência preferida de cada indivíduo, ao aumentarem a integração sensório-motor a nível cortical, estimulam a neuroplasticidade funcional e estrutural, potencializando conexões fronto-centroparietais/temporais complexas moduladoras da atividade da rede motora córtex-tálamo-cerebelo e fornecem ritmo externo que se sobrepõem a tal ritmo interno deficitário compensando falhas nos mecanismos de controle e geração do movimento rítmico e automático (CALABRÒ RS, et al., 2019).

Em estudo contando com 50 participantes, efeitos clínicos, cinemáticos e eletrofisiológicos do uso do RAS associado ao treino na esteira foram mensurados. Após 8 semanas de treinamento, o grupo com RAS apresentou melhora significativamente maior nas escalas de: avaliação funcional da marcha $(p<0,001)$; eficácia de queda de Tinetti ( $p<0,001)$; UPDRS $(p=0,001)$; e no índice geral de qualidade de marcha $(p<0,001)$ em relação ao grupo de treinamento sem RAS. Ademais, estes mecanismos foram corroborados pelas alterações eletroencefalográfica (CALABRÒ RS, et al., 2019).

Ratificando tais resultados, estudo com 60 pacientes com DP, demonstrou redução significativa no número de quedas e modificações em parâmetros-chave da marcha após treinamento com RAS. Apesar de ambos grupos (de treinamento com RAS continuo por 16 semanas e de controle de treinamento com RAS com

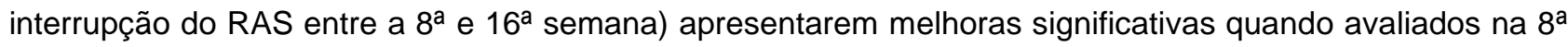
semana aumento da velocidade, comprimento de passada, cadencia e dorsiflexão bilateral dos tornozelos, e diminuição do índice de queda e medo de cair), na $16^{\underline{a}}$ semana diferenças significativas entre os grupos surgiram (THAUT MH, et al., 2019).

Essas diferenças incluem aumento no índice de queda $(p=0,0003)$, diminuição da velocidade $(p=0,0001)$, do comprimento de passada $(p=0,0001)$ e da dorsiflexão bilateral dos tornozelos no grupo controle. Após retorno do treino no grupo controle houve redução do número de quedas no mesmo, de forma que na $24^{a}$ semana diferenças entre os grupos já não eram significativas $(p<0,05)($ THAUT MH, et al., 2019).

Considerando que aumento da variabilidade do tempo de passada decorrente da perda de capacidade de produzir um ritmo estável aumenta risco de queda na DP, tais resultados ganham relevância na aplicação clínica do RAS. Ademais, o estudo demonstrou que a dorsiflexão apropriada do tornozelo durante a fase de balanço do ciclo da marcha pode ser mecanismo cinemático fundamental para prevenção de quedas, dado que o aumento da mesma correlacionou-se significativamente com declínio no índice de quedas e mudanças nos parâmetros cinemáticos da marcha (à exceção da cadência) (THAUT MH, et al., 2019).

Outrossim, efeitos da RAS dependem das habilidades de sincronização sensório-motora relativamente intactas para sustentar a sincronização de passos com pistas auditivas. Dada alta variabilidade individual de resposta aos efeitos da RAS, estudo com 14 participantes demonstrou que comprometimento de marcha mais grave, baixa variabilidade na sincronização e resposta imediata a mudança de estímulo associam-se a reposta positiva ao treinamento RAS (BELLA SD, et al., 2017).

Visando que baixa estabilidade e alto risco de quedas na DP estão relacionam-se a alterações cinemáticas típicas, Harrison EC, et al. (2020) realizou estudo com 35 participantes analisando efeitos cinemáticos das pistas auditivas externas e autogeradas (canto) sobre trajetória dos membros inferiores durante a marcha. Encontrou-se diferenças significativas em tais efeitos comparando respondedores e não respondedores à musicoterapia. Enquanto no grupo de respondedores houve aumento da amplitude geral de movimento (ADM) das articulações do quadril com ambos os tipos de pistas auditivas, aumento também da ADM no tornozelo com as pistas autogeradas (sugerindo maior eficácia das pistas autogeradas), e maiores excursões conjuntas na fase de apoio e de balanço, o grupo de não respondedores apresentou leve redução da ADM geral com as pistas auditivas (HARRISON EC, et al., 2020). 
Assim, postulou-se que a incapacidade de alteração cinemática a nível articular em não respondedores pode refletir inflexibilidade do sistema neuromuscular (possivelmente associado a idade avançada), e dificuldade de detectar a batida sonora e combiná-la com seus movimentos (talvez associada a menor experiência musical) (HARRISON EC, et al., 2020).

Aos efeitos benéficos da musicoterapia passiva no âmbito motor adicionam-se efeitos positivos na modulação emocional e cognitiva. Simplesmente ouvir música associa-se a ativação de diferentes circuitos anatômicos dopaminérgicos. Isto foi ratificado em estudo com 32 pacientes com DP no qual a associação de música rítmica com programa de reabilitação multimodal centrado na fisioterapia refletiu-se em melhorias estatisticamente significativas, tanto no valor global do PDQ-39 $(p<0,001)$, quanto nas subáreas de AVDs $(p=0,002)$, bem-estar emocional $(p=0,001)$, suporte social $(p=0,02)$, comunicação $(p=0,002)$ e desconforto corporal $(p=0,002)$, em comparação ao grupo controle submetido a mesma terapia de reabilitação sem música (FODOR DM, et al., 2021).

\section{Ambulosono}

Dois estudos avaliaram o uso da plataforma tecnológica GaitRemainder iPod, popularmente conhecido com Ambulosono, como opção terapêutica na DP, sendo método de treino de marcha com música baseada no uso de técnicas de condicionamento comportamental para estimular a neuroplasticidade e remodelar mecanismos compensatórios. Criando uma contingência entre os parâmetros de marcha e a reprodução de música, forma-se sistema de biofeedback simultâneo, que a reprodução musica depende do usuário atingir parâmetros de marcha pré-definidos, baseados em desempenho individual. Logo, ativam-se sistemas dopaminérgicos e não dopaminérgicos endógenos estimulando atenção, redes motoras e motivacionais (BURT J, et al., 2020).

Em estudo com 30 pacientes com DP o uso do Ambulosono mostrou-se viável e seguro. Com intuito de avaliar os efeitos do treino de marcha com música contingente na cognição e humor de pacientes com DP, participantes foram divididos em grupo de treinamento com Ambulosono e grupo controle de treino hibrido. Embora não tenham sido encontradas melhorias significativas nos parâmetros cognitivos, foram observadas diminuições significativas nos níveis de depressão e ansiedade em ambos grupos ao fim da intervenção $(p<0,05)$. Ainda, participantes eram altamente funcionais, de forma que o espaço para melhorias era limitado, sugerindo efeito de teto (BURT et al., 2020).

Respaldando tais resultados, Chomiak T, et al. (2017) demonstrou a eficácia do Ambulosono em melhorar automaticidade do passo em contextos de dupla-tarefa (DT). Postula-se que melhora global na automaticidade no grupo de intervenção $(p<0,01)$ esteja associada ao aumento da dopamina no putâmem posterior pelo feedback do Ambulosono e consequente deslocamento das habilidades motoras automatizadas para o estriado sensório-motor (CHOMIAK T, et al., 2017).

\section{Musicoterapia Ativa}

Musicoterapia Ativa (MT) foi avaliada quanto a seus potenciais efeitos positivos na cognição, qualidade de vida e sintomas motores em pacientes com DP em estudo envolvendo 25 participantes. Após 6 meses de sessões semanais de produção de música, canto e dança, detectou-se efeito positivo da MT no desempenho cognitivo e significativa melhora na qualidade de vida, especialmente em relação ao bem-estar emocional, no grupo de intervenção, contrastando com deterioração nas subescalas de desconforto corporal e mobilidade do PDQ-39 no grupo controle (SPINA E, et al., 2016).

Entretanto, tais resultados regrediram 6 meses após fim da intervenção sugerindo necessidade de manutenção da MT por período maior. Já sintomas motores, apesar de não terem apresentado alterações significativas ao fim da intervenção, houve deterioração nas pontuações do TUG entre o final da intervenção e 6 meses após, indicando possível efeito benéfico da MT na marcha e equilíbrio (SPINA E, et al., 2016).

Análise de 2 ensaios clínicos randomizados corrobora a pratica da dança como benéfica para pacientes com DP. Dança, além de agradável e envolvente, incorpora elementos-chaves do equilíbrio dinâmico, adaptação ao ambiente e estímulos externos derivados da música e do parceiro e estimulam redes de controle motor, aprimorando habilidades motoras e de equilíbrio. Em estudo com 20 participantes, no qual avaliou-se 
a viabilidade de protocolo de samba brasileiro em pacientes com DP, o grupo de dança apresentou melhora significativa no UPDRS $(p<0,001)$, escores de equilíbrio $(p=0,006)$, escores de mobilidade, atividades diárias, exame motor e avaliação de auto percepção de qualidade de vida associada a mobilidade $(p=0,019)$. (TILLMANN AC, et al., 2019).

Corroborando isto, em estudo com 90 participantes, benefícios da dança irlandesa foram comparados com o cuidado usual em pacientes com DP. Resultados demonstraram que o grupo de dança apresentou ganho, apesar de estatisticamente não significativo, na qualidade de vida em relação ao outro, ao mesmo tempo em que esse apresentou queda na resistência física. Além, houve tendência à significância na melhoria da UPDRS-3 ( $P=0.07)$ no grupo de dança em relação ao grupo controle (SHANAHAN J, et al., 2017).

Ademais, ensaios clínicos analisaram a eficácia do uso de pistas auditivas autogeradas (exercícios vocais e de canto) como ferramenta para melhorar marcha, voz, comunicação e qualidade de vida em pacientes com DP. Em estudo com 23 participante, Harrison EC, et al. (2018) comparou padrões de marcha destes sob 5 condições, incluindo canto. Caminhar enquanto cantava mostrou-se não ser tarefa excessivamente exigente do ponto de vista cognitivo, não desviando a atenção da tarefa principal e não afetando negativamente seu desempenho (como ocorreu com DT). A grande vantagem do canto ao caminhar foi a melhora significativa na variabilidade de marcha, marcador de desempenho geral desta intimamente relacionado com instabilidade e perda da automaticidade. Isto ganha relevância frente o aumento da variabilidade temporal e espacial dos parâmetros de marcha com as condições de pistas auditivas externas (HARRISON EC, et al., 2018).

Somando-se a tais resultados, estudo com 60 participantes demonstrou que estes utilizaram ambos tipos de pistas auditivas para melhorar cadência e comprimento de passada. Todavia, aumentou-se a variabilidade de marcha com uso das pistas externas, enquanto pistas internas (especialmente canto mental) diminuíramna (HARRISON EC, et al., 2020).

Outrossim, evidenciou-se que musicoterapia é eficaz em aprimorar inteligibilidade e parâmetros acústicos de fala. Distúrbios de fala e voz são comuns na DP, afetando cerca de $90 \%$ dos pacientes, e são extremamente resistentes às intervenções farmacológicas e fonoaudiologia. Debilidade de comunicação resulta em frustação, em pacientes e seus familiares, sendo que aqueles tendem a se afastar das conversas e atividades sociais, prejudicando seriamente sua qualidade de vida. Assim, cantar apresenta-se como intervenção envolvente, resultando em ampla ativação neural e usa muitas das estruturas e mecanismos da fala, exigindo aumento do suporte ventilatório, fonação alta e sustentada, variação de tom e articulação, intervindo em elementos prejudicados na DP (TAMPLIN J, et al., 2019).

Em estudo com 75 pacientes, intervenção terapêutica interdisciplinar baseada em canto, ParkinSong, resultou melhoras significativas na intensidade vocal $(p=0,018)$, pressão expiratória máxima $(p=0,032)$, e qualidade de vida relacionada à voz $(p=0,011)$ em comparação ao grupo controle. Ademais, houve efeito de dose $(p=0,011)$, uma vez que participantes do grupo de intervenção semanal aumentaram intensidade vocal mais do que pacientes do grupo de intervenção mensal (TAMPLIN J, et al., 2019).

Ratificando tais resultado, Han EY, et al. (2018) demonstrou que programa de canto terapêutico individual para DP foi capaz de aumentar significativamente Tempo Máximo de Fonação (MPT) ( $p=0,01$ ), e diminuir Índice de Desvantagem da Voz $(p=0,035)$ e Escala de Depressão Geriátrica (GDS) $(p=0,009)$ após a intervenção. Ademais, efeitos positivos no MPT, na qualidade de vida relacionada a voz e no GDS mantiveram-se após 6 meses de intervenção (HAN EY, et al., 2018).

\section{Método de terapia Ronnie Gardiner Rhythm and Music (RGRM)}

Pelo ensaio com 18 participantes, outra terapia alternativa promissora seria o uso do RGRM. Criado em 1973,este foca em exercícios que, através de ritmo e música, desafiam cognição e controle sensoriomotor para melhorar mobilidade e coordenação motora em pacientes com déficits neurológicos ( $\mathrm{POHL} P$, et al, 2013).

Entretanto, eficácia do método não encontrou apoio em estudo realizado com 46 pacientes com DP. Após programa de intervenção de 12 semanas, não houve diferenças significativas entre grupos para habilidade de dupla-tarefa e, apesar de ter existirem resultados positivos para Escala de Eficácia de Quedas $(p=0,001)$ e para o PDQ-39 $(p=0,005)$, não foram mantidos 3 meses após intervenção. Todavia, considerando-se que 
capacidade de aprender novas habilidades motoras complexas estavam comprometidas para muitos participantes, uma vez que $50 \%$ dos participantes apresentavam déficits cognitivos, pode ter contribuído para ausência de resultados significativos (POHL P, et al., 2020).

\section{CONSIDERAÇÕES FINAIS}

A musicoterapia mostrou-se eficaz como terapia adjuvante na Doença de Parkinson, com resultados positivos no manejo dos sinais e sintomas motores e não motores, além da melhora na qualidade de vida dos pacientes, sendo eficaz o uso de diversos métodos, em contextos de simples e dupla tarefa. Entretanto, há escassez de informações atuais tendo sido necessário expandir o recorte temporal para aumentar a abrangência. Ademais, estudos foram realizados com pequeno universo amostral, intervenções curtas, avaliando apenas efeitos em curto prazo, além da maioria analisar pacientes avaliados com DP de leve a moderada. Assim, para explorar melhor o tema, são necessários mais ensaios clínicos randomizados contando com musicoterapeuta, maior universo amostral e tempo de intervenção, avaliando resultados a longo prazo e pacientes com DP grave.

\section{REFERÊNCIAS}

1. BELLA SD, et al. Gait improvement via rhythmic stimulation in Parkinson 's disease is linked to rhythmic skills. Scientific Reports, 2017; 7(42005): 1-11.

2. BRASIL. Protocolo Clínico e Diretrizes Terapêuticas. 2010. Disponível em: http://www.portalarquivos.saude.gov.br/images/pdf/2014/abril/02/pcdt-doenca-parkinson republicado-livro-2010.pdf. Acessado em: 19 de julho de 2021.

3. BRUSCIA KE. Definindo Musicoterapia. $3^{\underline{a}}$ ed. Tradução de Marcos Leopoldino. Dallas: Barcelona Publischers, 2016; $1-5 p$.

4. BURT J, et al. The Effects of Music-Contingent Gait Training on Cognition and Mood in Parkinson Disease : A Feasibility Study. Neurorehabilitation and Neeural Repair, 2020; 34(1): 82-92.

5. CALABRÒ RS, et al. Walking to your right music : a randomized controlled trial on the novel use of treadmill plus music in Parkinson's disease. Journal of Neuroengineeringo and Rehabilitation, 2019; 16(1): 68-82.

6. CHOMIAK T, et al. A training approach to improve stepping automaticity while dual-tasking in Parkinson ' $s$ disease. Medicine, 2017; 96(5): 5934-5940.

7. CÔRTE B, LODOVICI NETO P. A musicoterapia na doença de Parkinson. Ciência \& Saúde Coletiva, 2009; 14(6): 2295-2304.

8. FODOR DM, et al. Music as Add-On Therapy in the Rehabilitation Program of Parkinson 's Disease Patients - A Romanian Pilot Study. Brain Science, 2021; 11(5): 569-568.

9. HAN EY, et al. Individual Therapeutic Singing Program for Vocal Quality and Depression in Parkinson's Disease. Journal of Movement Disorders, 2018; 11(3): 121-128.

10. HARRISON EC, et al. Mental Singing Reduces Gait Variability More Than Music Listening for Healthy Older Adults and People With Parkinson Disease. Journal of Neurologic Physical Therapy, 2020; 43(4): 204-211.

11. HARRISON EC, et al. The Feasibility of Singing to Improve Gait in Parkinson Disease. Gait e Posture, 2018; 53(1): 224-229.

12. HU B, et al. Walking with music is a safe and viable tool for gait training in parkinson's disease: The effect of a 13-week feasibility study on single and dual task walking. Parkinson's Disease, 2010; 2010 (1): 1-9.

13. MORATELLI J, et al. Feasibility of a Brazilian samba protocol for patients with Parkinson 's disease : a clinical nonrandomized study. Arquivos de neuropsiquiatria, 2019; 78(1): 13-20.

14. OLIVEIRA MF, et al. Musicoterapia como ferramenta terapêutica no setor da saúde: uma revisão sistemática. Revista da universidade vale do rio verde, $2014 ; 12(2): 871-878$.

15. POHL P, et al. Group-based music intervention in Parkinson 's disease - findings from a mixed-methods study. Clinical Rehabilitation, 2020; 34(4): 533-544.

16. POHL P, et al. The Ronnie Gardiner rhythm and music method-A feasibility study in parkinson's disease. Disability and Rehabilitation, 2013; 35(26): 2197-2204.

17. SANTOS FR, CORONAGO VM. Uso da Musicoterapia como Terapia Alternativa no Tratamento da Doença de Parkinson. Revista De Psicologia, 2017; 11(35): 341-360.

18. SHANAHAN J, et al. Dancing for Parkinson Disease: A Randomized Trial of Irish Set Dancing Compared With Usual Care. Archives of Physical Medicine and Rehabilitation, 2017; 98(9): 1744-1751.

19. SIHVONEN AJ, et al. Music-based interventions in neurological rehabilitation. The Lancet Neurology, 2017; 16(8): 648660.

20. SPINA E, et al. Music Therapy for Motor and Nonmotor Symptons of Parkinson's Disease: A prospective, Randomized, Controlled, Single-Blinded Study. Journal of the American Geriatrics Society, 2016; 64(9): 36-39.

21. TAMPLIN J, et al. ParkinSong: A Controlled Trial of Singing- Based Therapy for Parkinson's Disease. Neurorehabilitation ans Neural Repair, 2019; 33(6): 453-463.

22. THAUT MH, et al. Rhythmic auditory stimulation for reduction of falls in Parkinson ' $s$ disease : a randomized controlled study. Clinical Rehabilitation, 2019; 33(1): 34-43.

23. VALCARENGHI RV, et al. The daily lives of people with Parkinson's disease. Revista brasileira de enfermagem, 2018; 71(2): 272-279.

24. WERNECK AL. Doença de Parkinson: Etiopatogenia, Clínica e Terapêutica. Revista Hospital Universitário Pedro Ernesto, 2010; 9(1): 10-19. 